Modeling the Structural Response from a Propagating High Explosive Using Smooth Particle Hydrodynamics

J. Margraf

June 15, 2012 
This document was prepared as an account of work sponsored by an agency of the United States government. Neither the United States government nor Lawrence Livermore National Security, LLC, nor any of their employees makes any warranty, expressed or implied, or assumes any legal liability or responsibility for the accuracy, completeness, or usefulness of any information, apparatus, product, or process disclosed, or represents that its use would not infringe privately owned rights. Reference herein to any specific commercial product, process, or service by trade name, trademark, manufacturer, or otherwise does not necessarily constitute or imply its endorsement, recommendation, or favoring by the United States government or Lawrence Livermore National Security, LLC. The views and opinions of authors expressed herein do not necessarily state or reflect those of the United States government or Lawrence Livermore National Security, LLC, and shall not be used for advertising or product endorsement purposes.

This work performed under the auspices of the U.S. Department of Energy by Lawrence Livermore National Laboratory under Contract DE-AC52-07NA27344. 


\section{Modeling the Structural Response from a Propagating High Explosive Using Smooth Particle Hydrodynamics}

\section{Finite Element Codes}

This report primarily concerns the use of two massively parallel finite element codes originally written and maintained at Lawrence Livermore National Laboratory. ALE3D is an explicit hydrodynamics code commonly employed to simulate wave propagation from high energy scenarios and the resulting interaction with nearby structures. This coup led response ensures that a structure is accurately applied with a blast loading varying both in space and time. Figure 1 illustrates the radial outward propagation of a pressure wave due to a center detonated spherical explosive originating from the lower left.
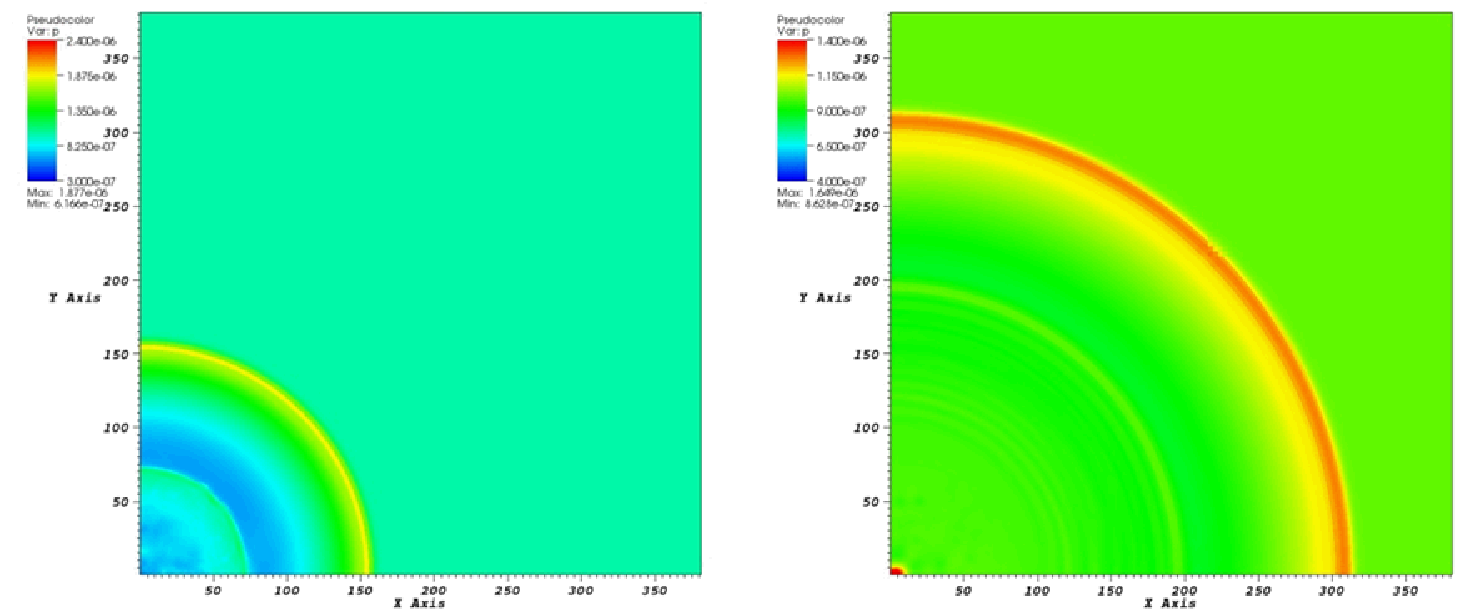

Figure 1: ALE3D Spherical Charge Wave Propagation 
The radial symmetry seen in this scenario is lost when instead a cylindrical charge is detonated. Figure 2 indicates that a stronger, faster travelling pressure wave occurs in the direction of the normal axis to the cylinder.
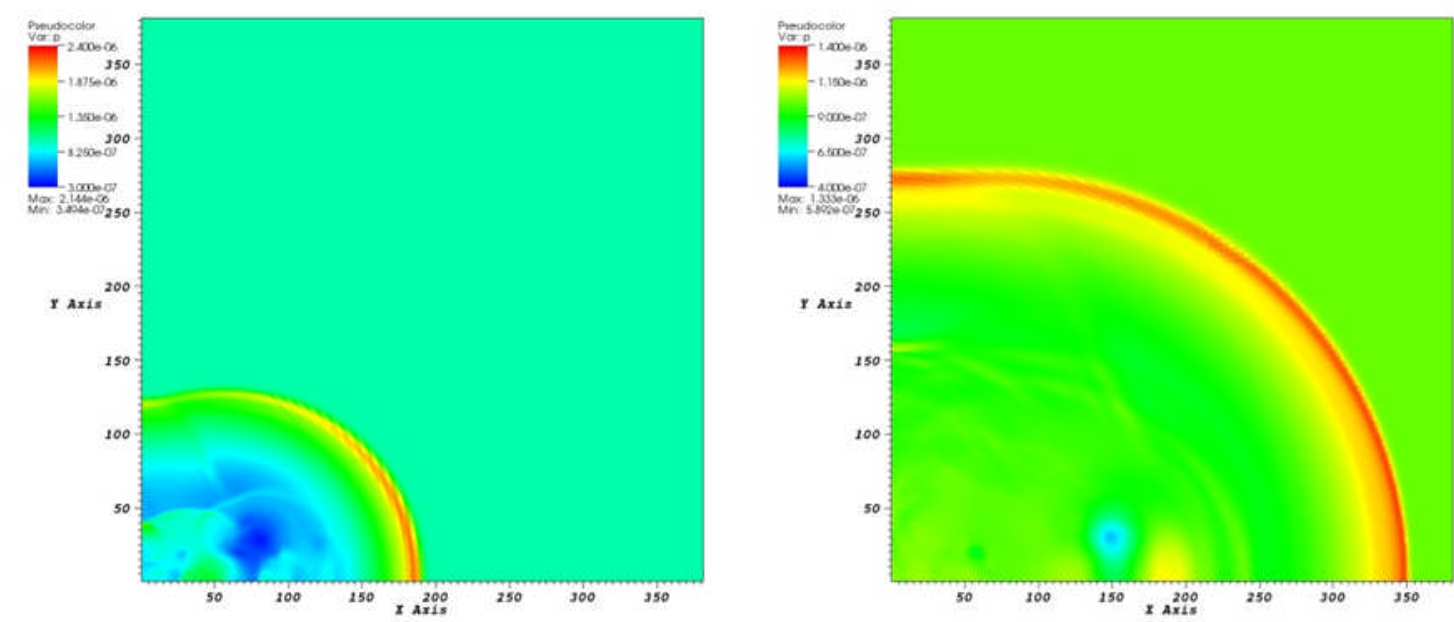

Figure 2: ALE3D Cylindrical Charge Wave Propagation

The ALE3D name is derived because of the use of arbritrary-Lagrange-Eulerian elements in which the mesh is allowed to advect; a process through which the mesh is modified to alleviate tangling and general mesh distortion often caused by high energy scenarios. The counterpart to an advecting element is a Lagrange element, whose mesh moves with the material. Ideally all structural components are kept Lagrange as long as possible to preserve accuracy of material variables and minimize advection related errors. Advection leads to mixed zoning, so using structural Lagrange elements also improves the visualization when post processing the results. A simplified representation of the advection process is shown in Figure 3.

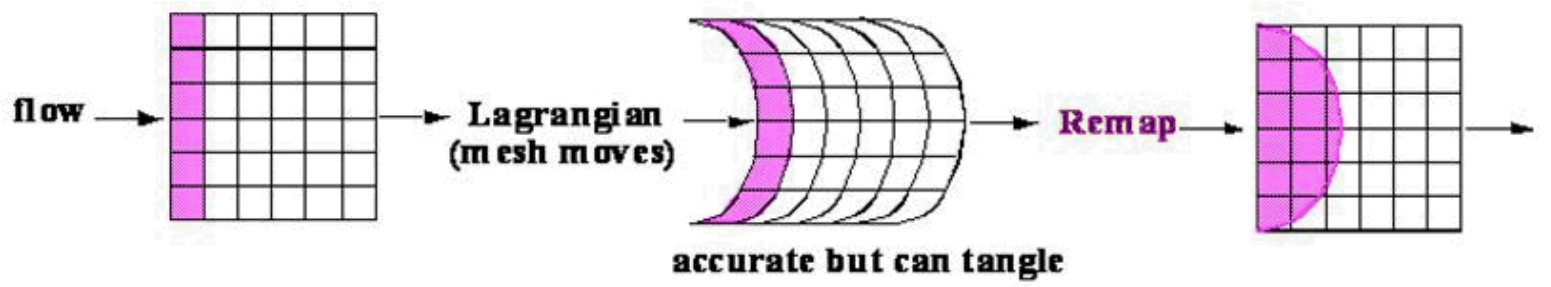

Figure 3: ALE3D Advection Process

First the mesh is distorted due to material motion during the Lagrange step. The mesh is then shifted to an idealized and less distorted state to prevent irregular zones caused by the Lagrange motion. Lastly, the state variables are remapped to the elements of the newly 
constructed mesh. Note that Figure 3 represents a purely Eulerian mesh relaxation because the mesh is relocated back to the pre-Lagrange position. This is the case when the material flows through a still mesh. This is not typically done in an ALE3D analysis, especially if Lagrange elements exist. Deforming Lagrange elements would certainly tangle with an Eulerian mesh eventually. The best method in this case is to have an advecting mesh positioned as some relaxed version of the pre and post Lagrange step; this gives the best opportunity of modeling a high energy event with a combination of Lagrange and ALE elements.

Dyna3D is another explicit dynamic analysis code, ParaDyn being the parallel version. ParaDyn is used for predicting the transient response of three dimensional structures using Largrangian solid mechanics. Large deformation and mesh tangling is often resolved through the use of an element deletion scheme. This is useful to accommodate component failure, but if it is done purely as a means to preserve a useful mesh it can lead to problems because it does not maintain continuity of the material bulk response. Whatever medium exists between structural components is typically not modeled in ParaDyn. Instead, a structure either has a known loading profile applied or given initial conditions. The many included contact algorithms can calculate the loading response of materials if and when they collide. A recent implementation of an SPH module in which failed or deleted material nodes are converted to independent particles is currently being utilized for a variety of spall related problems and high velocity impact scenarios. Figure 4 shows an example of a projectile, given an initial velocity, and how it fails the first plate which generates SPH particles which then interact with and damage the second plate.

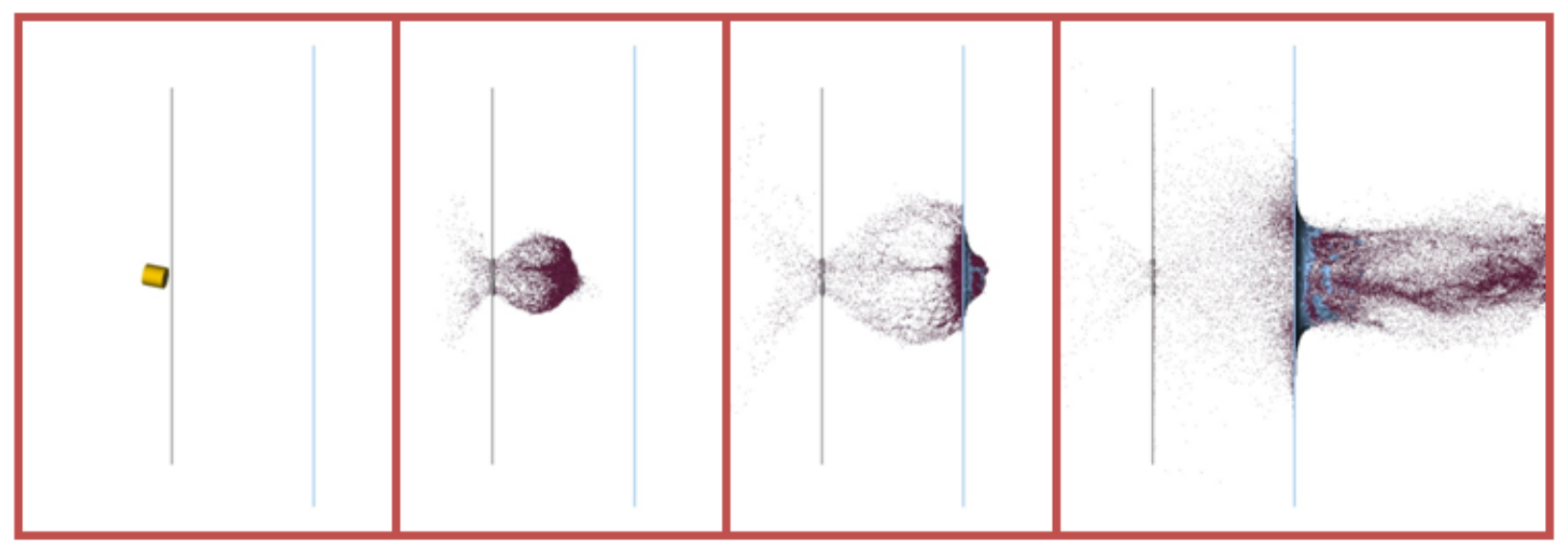

Figure 4: ParaDyn SPH Example 


\section{Motivation}

Subjecting complex geometrical features to shock loading can be problematic for ALE3D simulations. The need for Lagrangian structural elements, surrounded by advecting elements containing high velocity materials can cause a number of mesh related issues resulting in dramatic lowering of time steps or simulation crashes. Figure 5 depicts an example ALE3D simulation in which a material motion tends to drag the mesh downward and around the Lagrange nodes. This is acceptable in this scenario, but it highlights the potential difficulties when a variety of Lagrangian materials are present in the model.

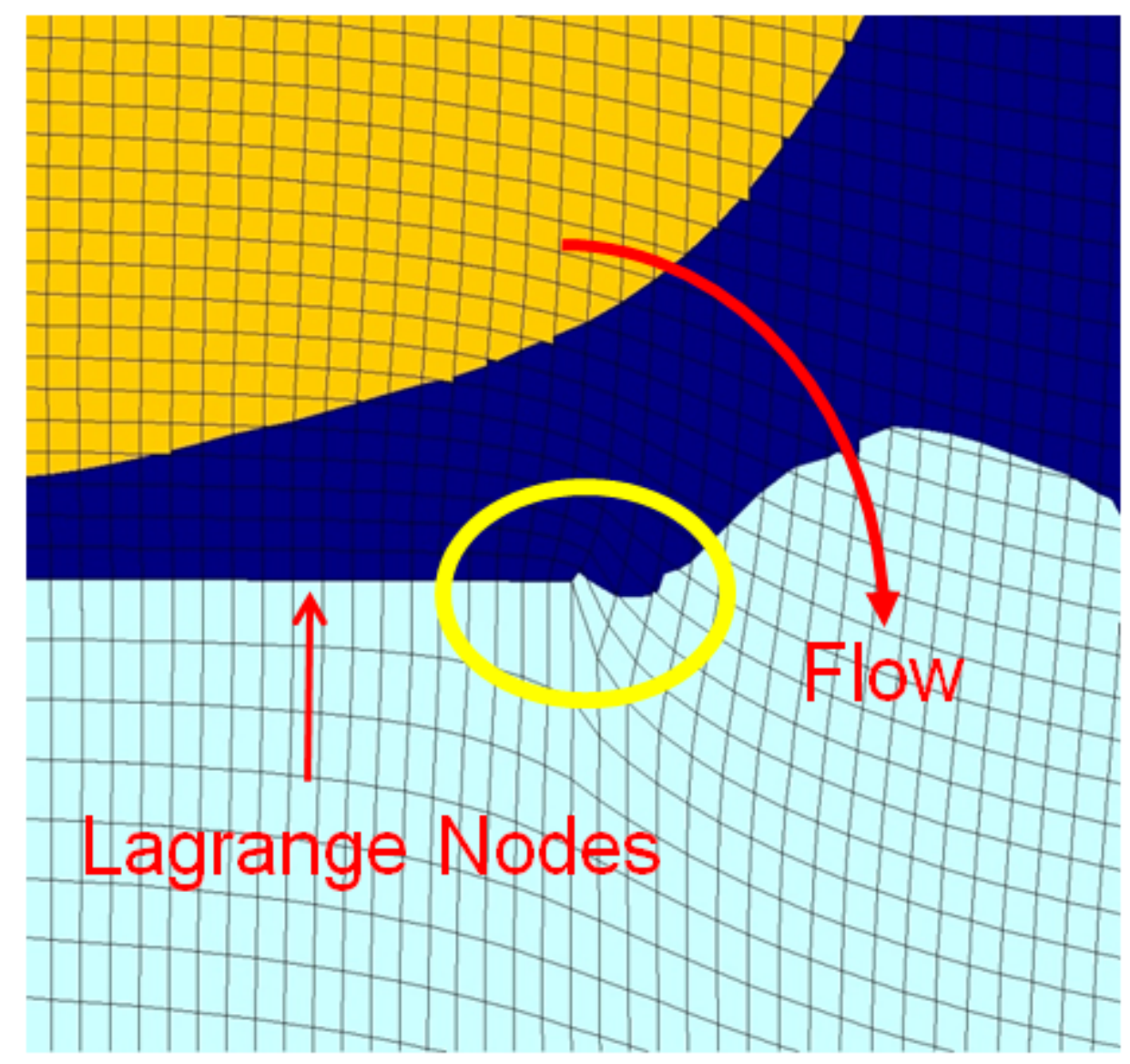

Figure 5: Potential ALE3D Mesh Tangling Issues

Excruciating attention to detail has been made to modeling a specific structure with intricate interconnecting components for a current project. It is impractical to analyze a blast scenario for this model in ALE3D for the reasons mentioned above. This model was built for ParaDyn with a simplified loading approach and has been utilized with a significant amount of success.

The conceived plan was a handshake method between ALE3D and ParaDyn. ALE3D will be executed for a similar yet less detailed structure where the major surfaces are loaded by the 
blast wave. These surfaces deform but do not fail. The blast pressures are collected for the surface locations and mapped to the corresponding surfaces in the ParaDyn model. The ParaDyn analysis loads the detailed structure based on the calculated pressure time histories, and ParaDyn handles the response by using the contact algorithms and eroding elements upon failure, which is ideal because the element no longer contributes structurally. While the internal components are present, thus adding stiffness of the global structure, the big assumption is that they are not being loaded directly by the explosive. This method has provided useful, insightful, and a wide range of predictive results.

However, by not coupling every structural component to the ALE3D analysis this technique is not immune to scrutiny. It is unknown if reflections or other fundamental interactions with the intermediate surfaces substantially influences the pressures applied to the major surfaces. It is also unknown if the damage to these components in tandem with the damage to the primary surface is negligible to the overall result or not. Therefore a fully coupled approach is desired, if at least for verification of the simplified method already in use.

The preferred method to accomplish this at present is a meshless method. The Smooth Particle Hydrodynamics module in ParaDyn will be employed, not as a representation of failed or eroded structural material, but as an expanding high energy gas cloud to fully represent the explosive blast wave. This capability will be examined in detail for a simpler surrogate model before it is attempted on the large model described earlier in this section.

\section{Smooth Particle Hydrodynamics}

Physical model behavior is predicted in Smooth Particle Hydrodynamics (SPH) by representing the material with a network of discrete particle elements. These particles are free to move while interacting with neighboring particles and other ordinary finite elements. These interactions are weighted according to the current particle separation at a current time step. In Dyna3D a particle is initialized when all adjacent elements have failed, and along with position, a particle inherits conditions such as pressure, energy, mass, and velocity.

When an element fails and SPH particles are created, momentum is conserved and the material equation of state is maintained. Because the represented material has failed, the particles have no strength thus no constitutive behavior is necessary. The density of a particle is a function of the weighted summation of surrounding particle masses. The communication radius or the smoothing length is roughly 1 to 1.5 times the average particle separation. Bounds can be set on both the smoothing length and the number of neighbors and may need to be modified in certain applications in order to get smooth particle communication. Note that the particle density can change dramatically with significant material expansion, perhaps driving the need for future implementation of particle birth methods. 


\section{Surrogate Model}

The large detailed structure is not reasonable for debugging purposes; therefore proof of capability will be tested on a scaled representation of it. The surrogate model inherits the material type, thickness, mesh resolution, and charge standoff of the detailed model. Figure 6 shows the two foot by two foot sheet of 0.071 " thick 2024-T3 aluminum built with $0.125^{\prime \prime}$ shell elements with three through thickness integration points. An explosive charge is placed in line with the center of the plate at a standoff of four inches (surface to surface), and the plate is fixed along the edges (translational degrees of freedom).

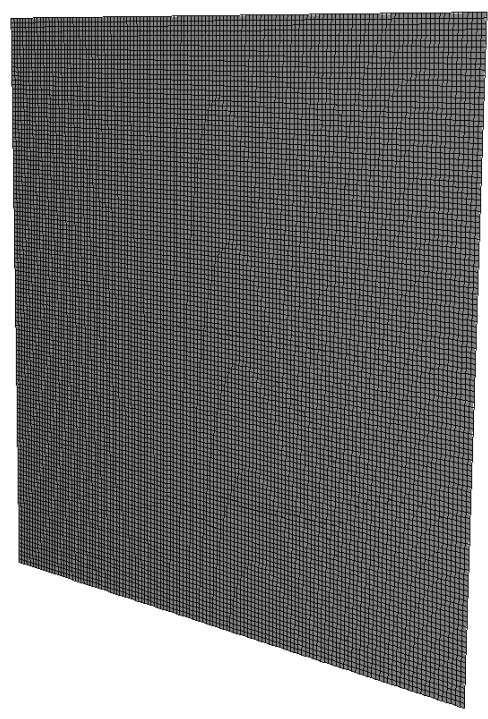

Figure 6: Surrogate Model

Documented properties for 2024-T3 aluminum using a kinematic/isotropic elastic plastic material model are shown in Table 1.

Table 1: 2023-T3 Aluminum Material Properties

\begin{tabular}{|c|c|c|c|c|c|c|}
\hline Thickness & Density & $\begin{array}{c}\text { Young's } \\
\text { Modulus }\end{array}$ & $\begin{array}{c}\text { Poisson's } \\
\text { Ratio }\end{array}$ & $\begin{array}{c}\text { Yield } \\
\text { Stress }\end{array}$ & $\begin{array}{c}\text { Tangent } \\
\text { Modulus }\end{array}$ & $\begin{array}{c}\text { Failure } \\
\text { EPS }\end{array}$ \\
\hline (in) & $(g / c c)$ & $($ Mbar) & $(-)$ & $($ Mbar) & (Mbar) & $(-)$ \\
\hline 0.071 & 2.768 & $\begin{array}{c}6.345 \mathrm{E}- \\
01\end{array}$ & 3.3 & $3.621 \mathrm{E}-03$ & $\begin{array}{c}1.138 \mathrm{E}- \\
02\end{array}$ & $\begin{array}{c}1.43 \mathrm{E}- \\
01\end{array}$ \\
\hline
\end{tabular}

Due to the simplicity of this model, the structural response from this charge weight and standoff is easily predicted with ALE3D. This result will be considered the real solution (no experiments were conducted), the control variable that SPH will try to reproduce. 
The ALE3D simulation serves another purpose other than validation tool. The state variables of the explosive products and the surrounding air will be imported to ParaDyn to properly define the initial status of the particles. Because the aluminum plate in ParaDyn is initially static, it is important that the ALE3D values be exported before there is any interaction with the structure. It is also important that this be done as late as possible as to allow ALE3D to simulate the explosive detonation. This is a serious issue for problems with closer standoffs because SPH is not designed to handle the chemistry involved in releasing the energy of detonation. Figure 7 shows a pressure plot from ALE3D along with the mapped pressures to the SPH particles.

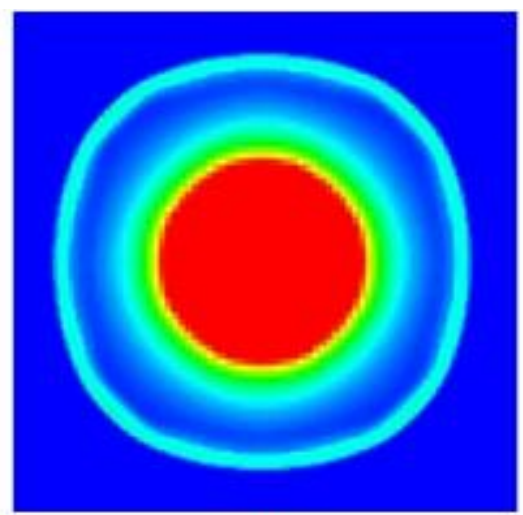

(a)

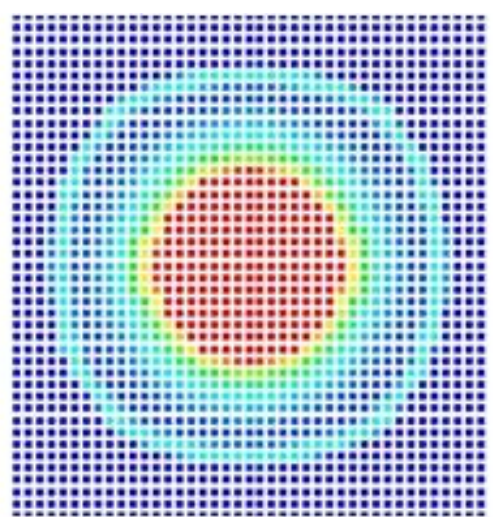

(b)

Figure 7: Expanding Pressure Wave, (a) ALE3D, (b) Mapped to Paradyn SPH

A block of many equally sized solid elements are meshed for ParaDyn with the intention of each node becoming an SPH particle. Because an SPH particle is born from the nodes of deleted elements, a trick is applied in the ParaDyn input deck to simultaneously set element failure for a material based on relative volume, and apply an initial relative volume above this limit. This causes all of these solid elements to erode at time zero and the SPH module to activate. Automatic contact will handle all of the interactions after the particles inherit the values from the ALE3D simulation. Figure 8 shows an example scenario of the early stage particle expansion. 


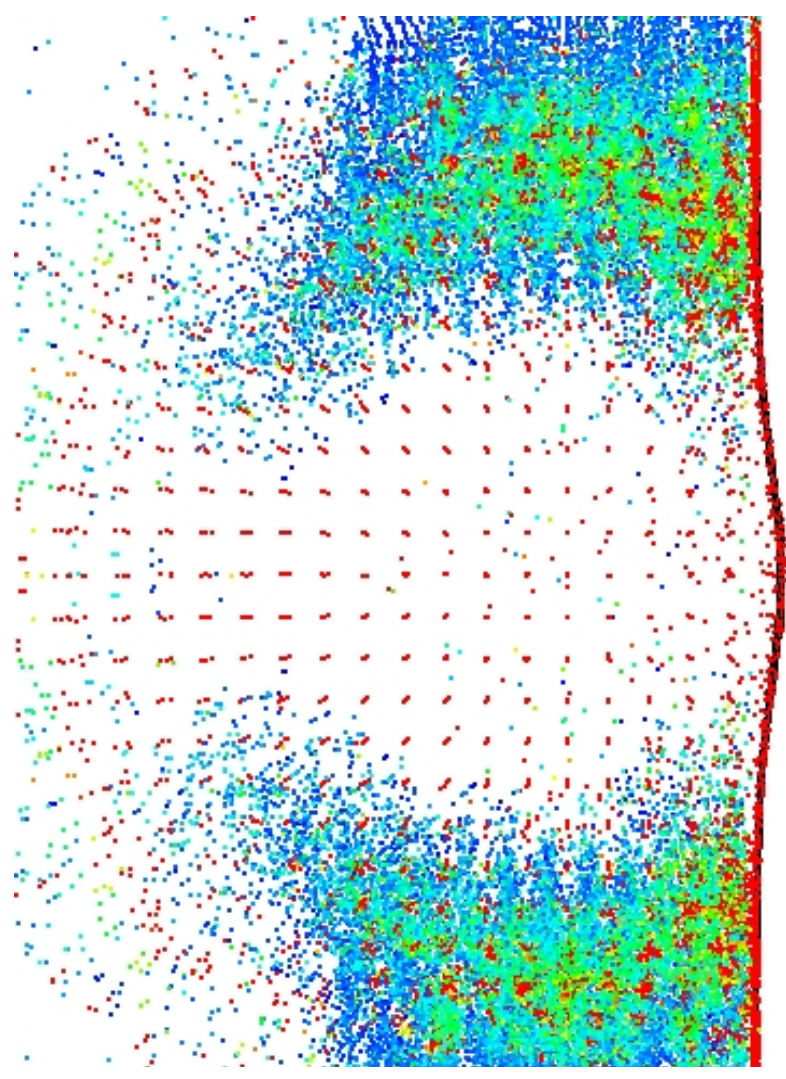

Figure 8: Early Stage SPH Particle Expansion

\section{Initial Inconsistent Strain Results}

Initial SPH calculations yielded unexpected results. The plate properly responds to the blast as the particles push against the plate, however the skin is tearing in the SPH model while it does not in the ALE3D analysis. Because strain is the failure criterion for this model, a closer examination of the strain plots shows that the ParaDyn simulation has an extremely scattered distribution in comparison to ALE3D (Figure 9). 

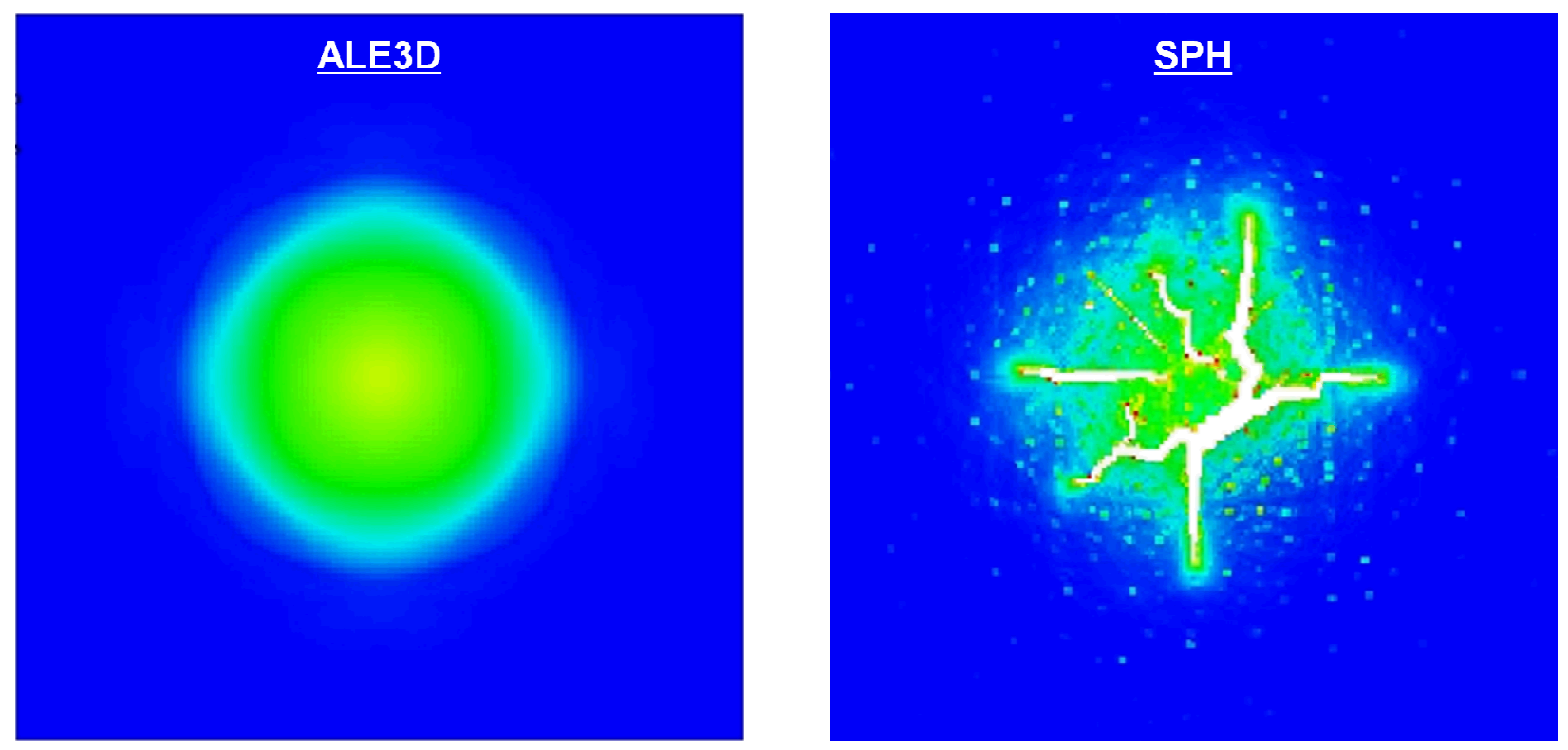

Figure 9: ALE3D and Paradyn SPH Strain Distribution Comparison

The localized strains can typically escalate above the failure threshold and cause a failed element. As the plate is loaded this is enough to form a rip. Figure 9 indicates that several rips have formed in this manner. This load scattering is undesired. The particles are behaving like a bunch of individual projectiles striking the plate whereas the goal is to replicate a smooth and continuous wave. Notice also that while this model has quarter symmetry, the SPH result does not. This could have to do with round-off errors or machine epsilon or some other randomness in the code and may require later investigation.

\section{A Study on SPH Particle Spacing}

The suspect results are the precursor to the study on the size of the particle spacing relative to the resolution of the structural elements. Simply put, an insufficient number of particles for every structural element may be the root cause of the scattered strain pattern.

Because the initial spacing of the SPH particles is determined by the mesh resolution of the SPH cloud build for Paradyn, it can easily be modified by changing the mesh of this initial cloud. The initial attempt uses five millimeter particle spacing (157\% compared to the one eighth inch structure mesh resolution). Scenario two will use approximately 4 millimeter spacing (125\% compared to the one eighth inch structure resolution). Scenario three will have the same spacing as the structure so that the nodes initially in line with one another. Figure 10 shows the three various initial SPH resolutions with the structure mesh in the background so an idea of the relative spacing differences can be visualized. 


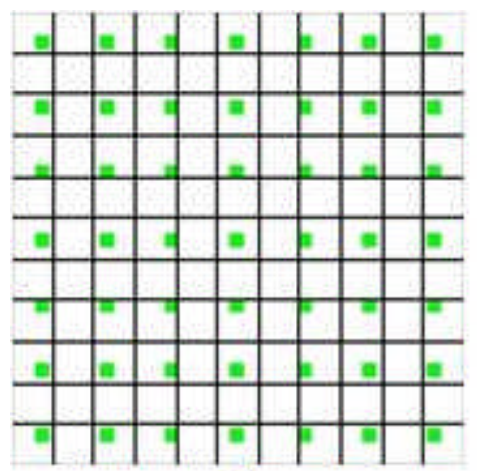

(a)

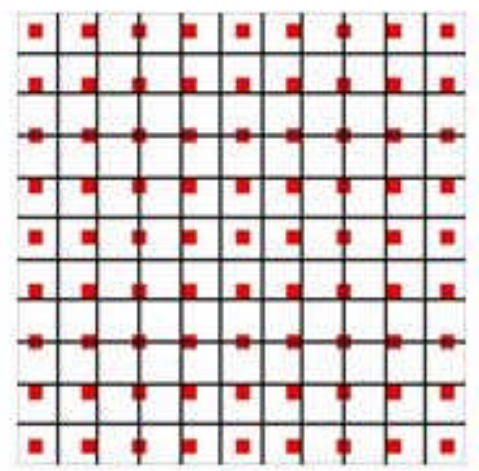

(b)

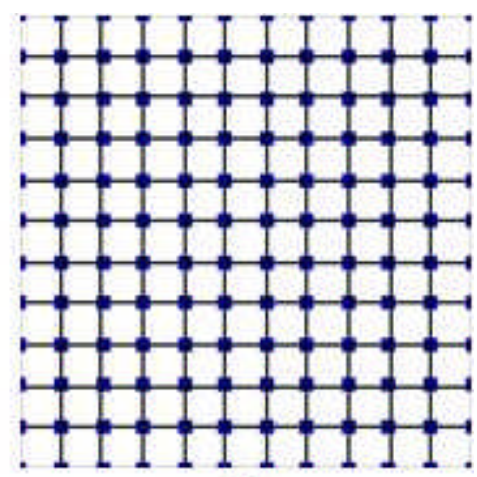

(c)

Figure 10: SPH Resolution Study, (a) Case 1: 5mm, (b) Case 2: 4mm, (c) Case 3: 3mm

All three cases contain 37,000 shell elements. Case 1 contains roughly $560,000 \mathrm{SPH}$ particles, is computed on 16 processors and makes it to 500 microseconds in six hours of run time. Case 2 contains just over a million SPH particles and on 32 processors the analysis made it to 800 microseconds in 9 hours of run time. Note that the added cost of running Case 2 as opposed to Case 1, while not small, is manageable. However, case 3 has about 2.5 million particles by using 64 processors the code calculated up to 300 microseconds in 15 hours. This scenario is prohibitively slow in comparison to the former simulations.

Figure 11 shows the three SPH strain distribution plots for comparison with the ALE3D approach at 100 microseconds. The ALE3D simulation shows a smooth and compact range of strain accumulation, while the SPH Case 1 shows a larger affected area of scattered strain spots that was seen before. A few hot spots of strain can be seen, the yellow and red elements prematurely approaching failure. The strain progressively refines to the spatial distribution to the ALE3D case as the SPH spacing is reduced. 


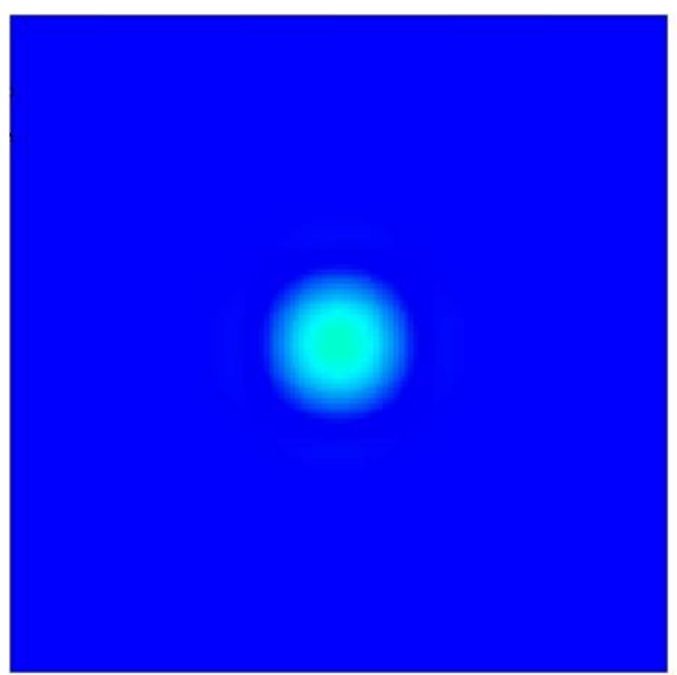

(a)

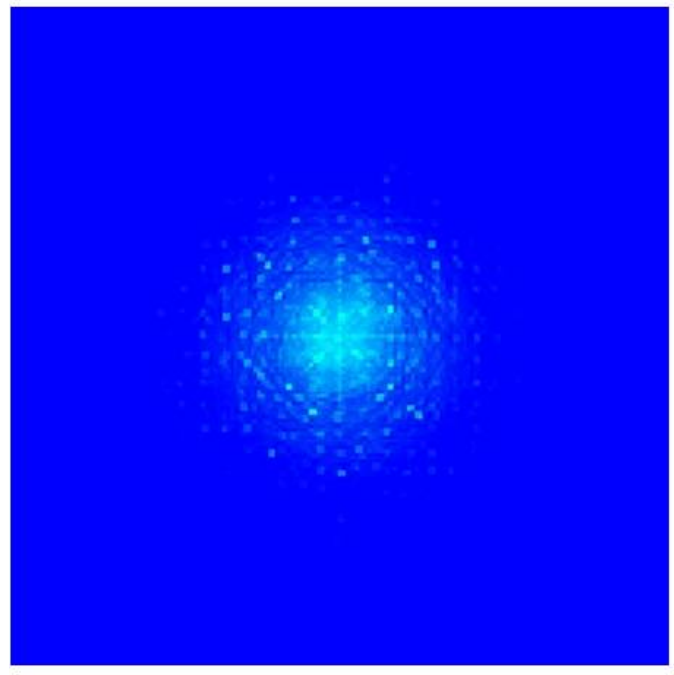

(c)

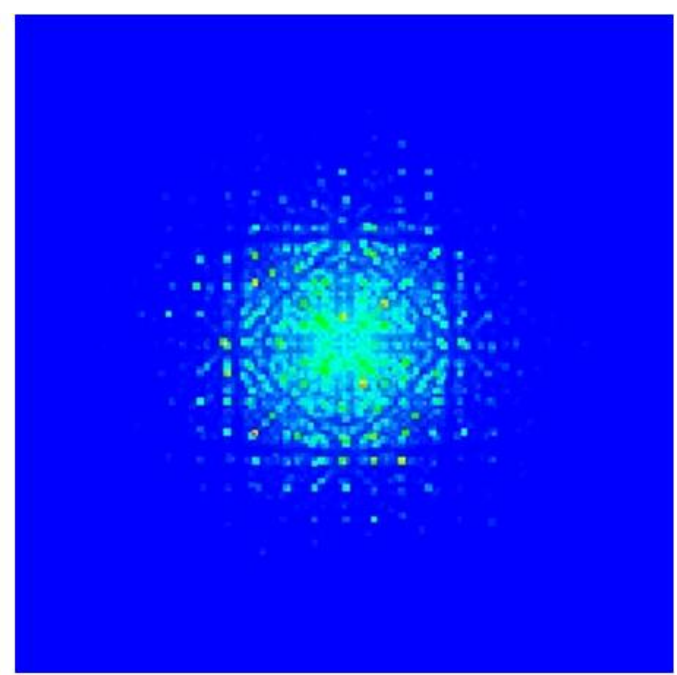

(b)

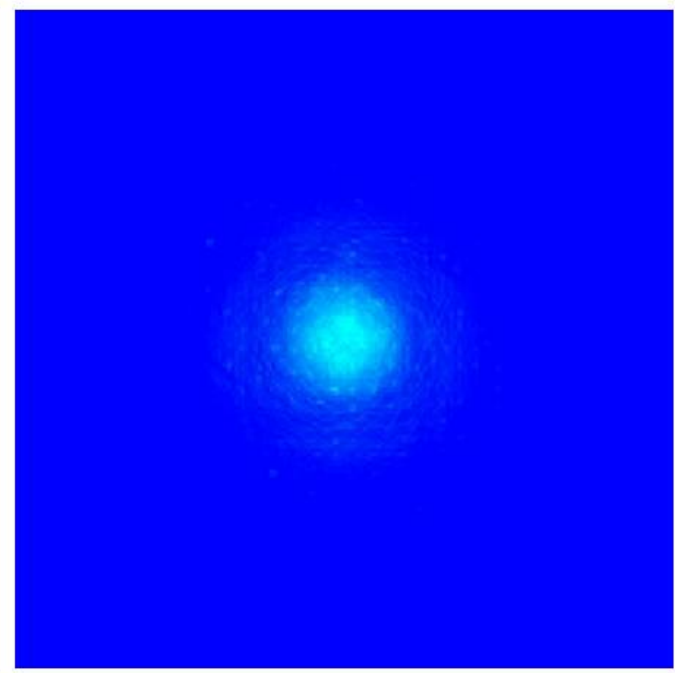

(d)

Figure 11: Strain Distributions at 100 microseconds, (a) ALE3D, (b) 5mm SPH Resolution, (c) $4 \mathrm{~mm}$ SPH Resolution, (d) 3mm SPH Resolution

At 150 microseconds the low resolution SPH has now failed some of the shell elements and the aluminum is now tearing (Figure 12). This fallacy has been avoided with the higher resolution SPH particle approaches. 


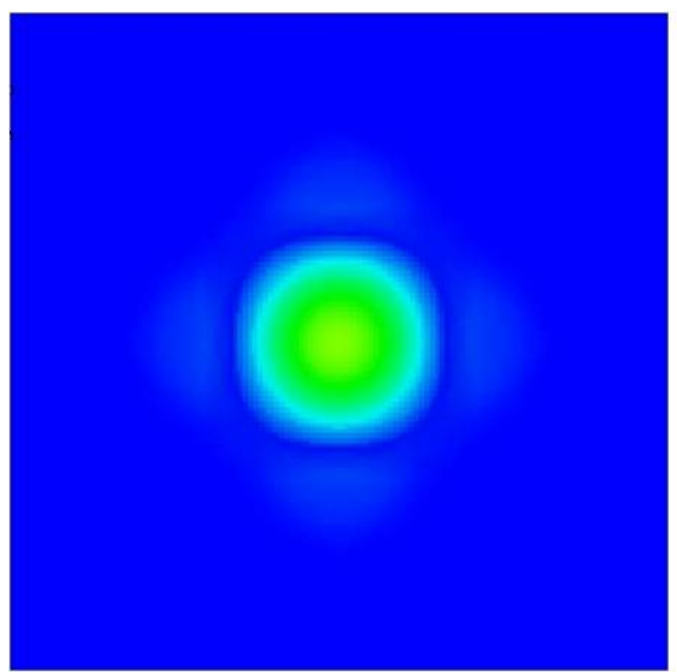

(a)

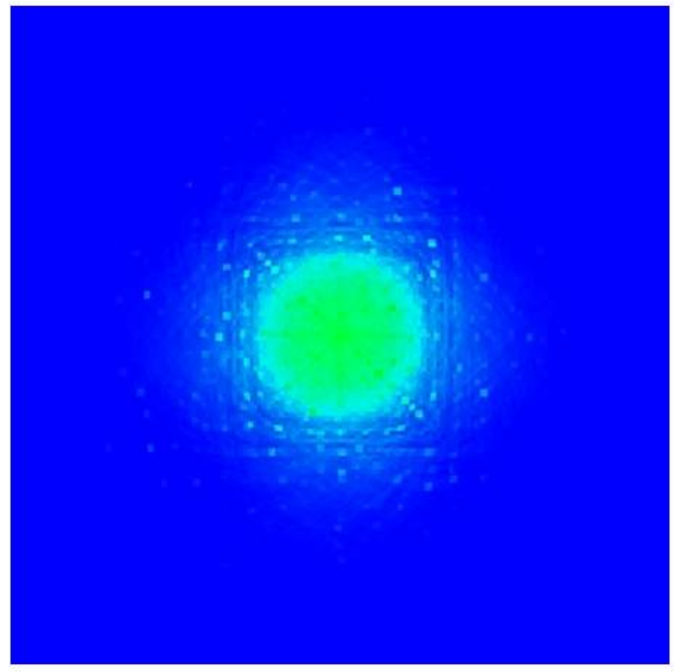

(c)

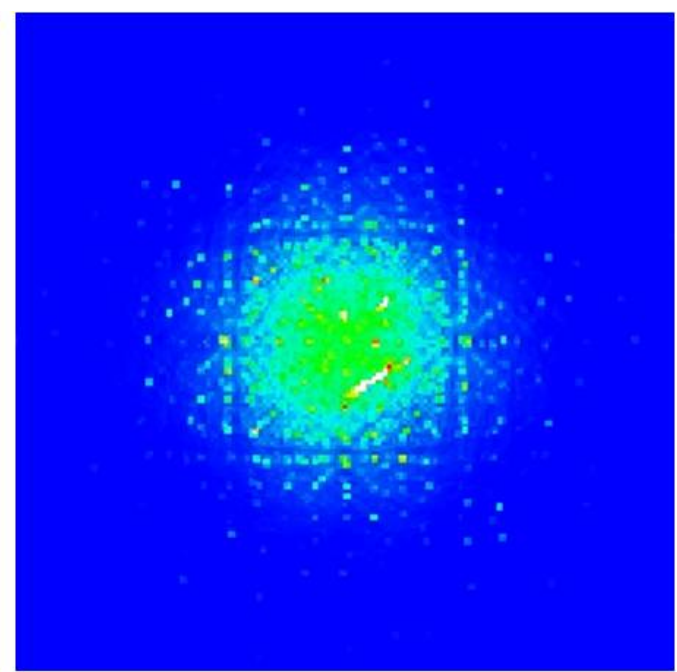

(b)

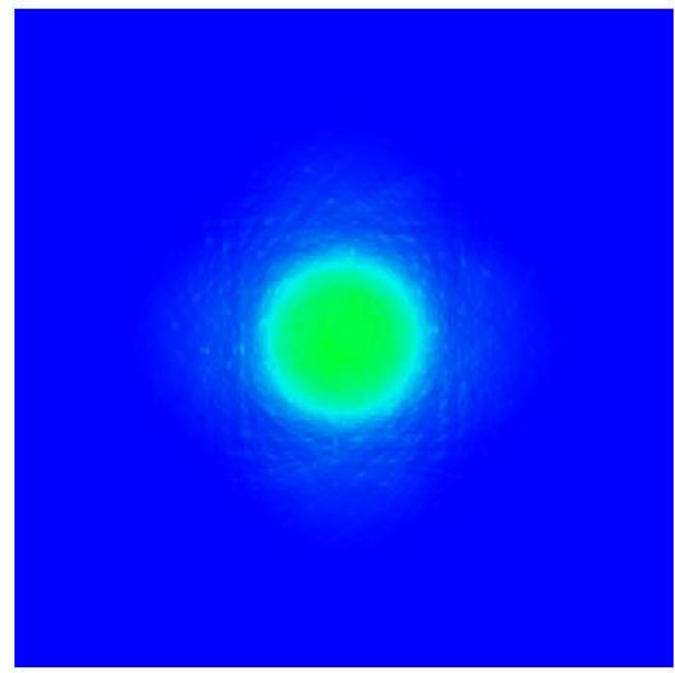

(d)

Figure 12: Strain Distribution at 150 microseconds, (a) ALE3D, (b) 5mm SPH Resolution, (c) $4 \mathrm{~mm}$ SPH Resolution, (d) 3mm SPH Resolution

By 300 microseconds the plate using the low resolution SPH has began to open up, while neither of the other two scenarios have failed elements and likely won't later because the heaviest loading has now passed (Figure 13). There is negligible difference between case two and case three as they well replicate the spatial distribution of the strain behavior, although neither has quite generated the peak strain in the center. The issue with scattered and localized strain behavior using SPH for blast dynamics has been resolved for this model. 


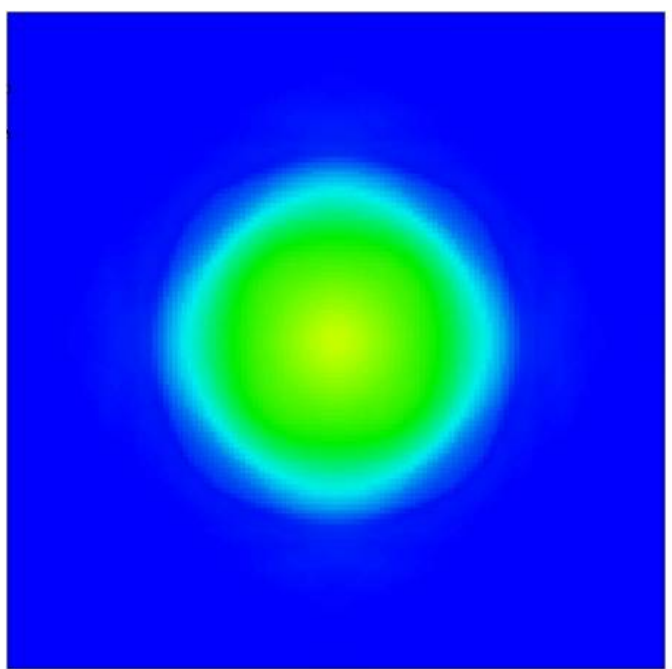

(a)

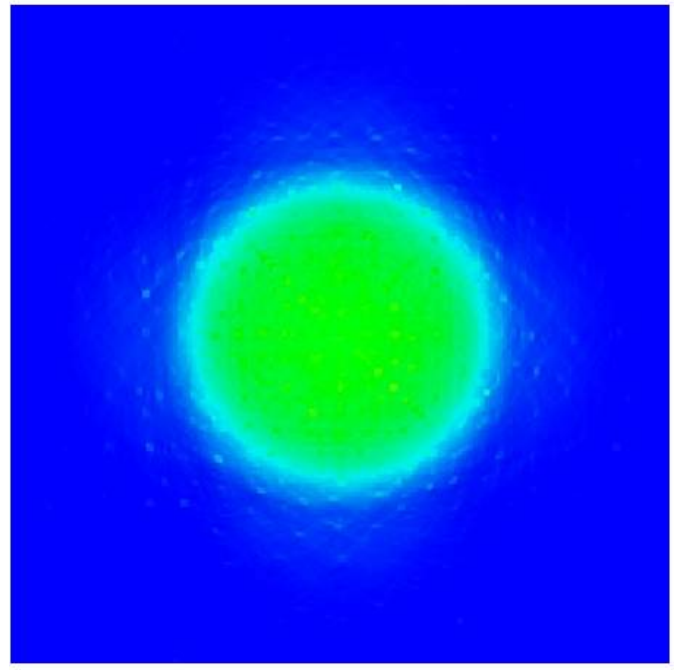

(c)

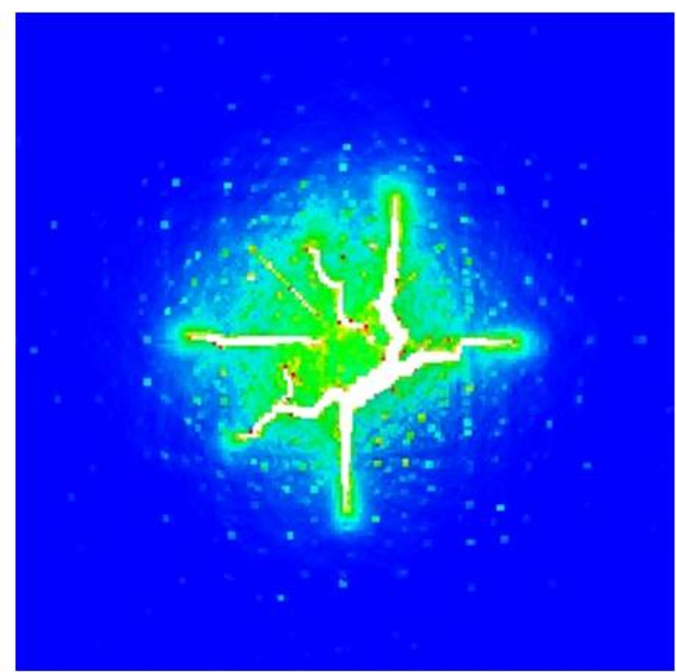

(b)

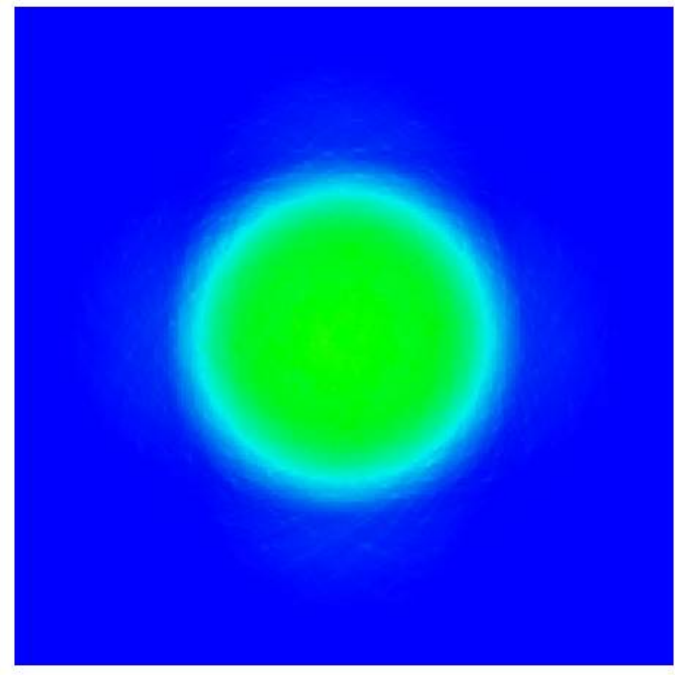

(d)

Figure 13: Strain Distribution at 300 microseconds, (a) ALE3D, (b) 5mm SPH Resolution, (c) $4 \mathrm{~mm}$ SPH Resolution, (d) 3mm SPH Resolution 


\section{ALE3D and SPH Displacement Comparisons}

Plate failure is not predicted for this combination of plate material and geometry, charge standoff, and charge weight. The strain was important only to alleviate the initial problems with untimely failure. In spite of this the key metric for comparison in this study has always been displacement. Because Case 3 is considered prohibitively expensive with minimal benefit compared to Case 2, it has been decided that Case 2 produces the optimum combination of accuracy and efficiency.

Figure 14 shows the displacement comparison between ALE3D and Paradyn SPH with 4 millimeter initial particle spacing at 250 microseconds. Displacement is beginning at the center and expanding outwards. Good comparison is seen between the two plots.

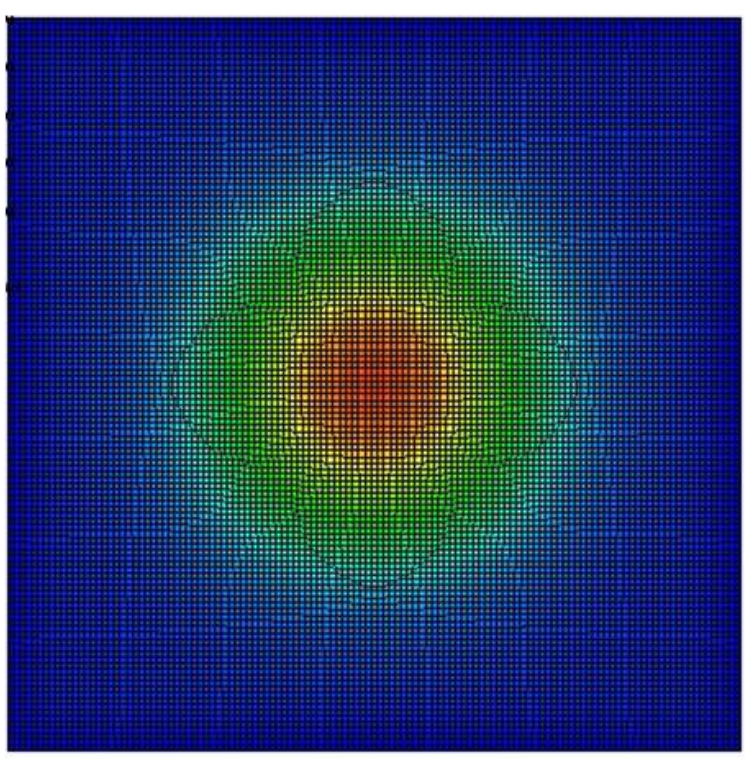

(a)

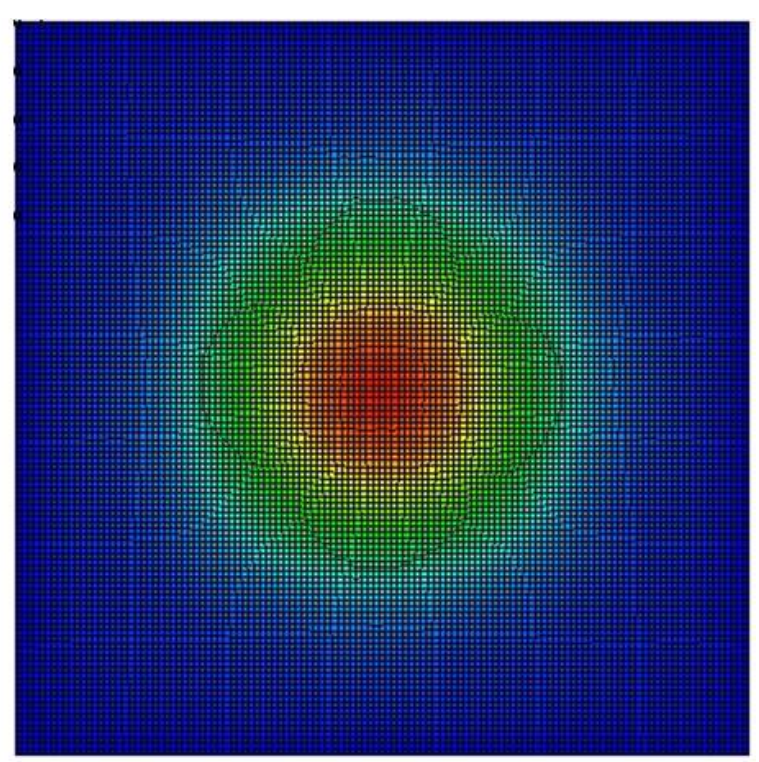

(b)

Figure 14: Dipslacement Contour Comparison at 250 microseconds, (a) ALE3D, (b) Paradyn SPH 
At 500 microseconds displacement is extending to the fixed boundary and good spatial comparisons can be made between the two codes (Figure 15).

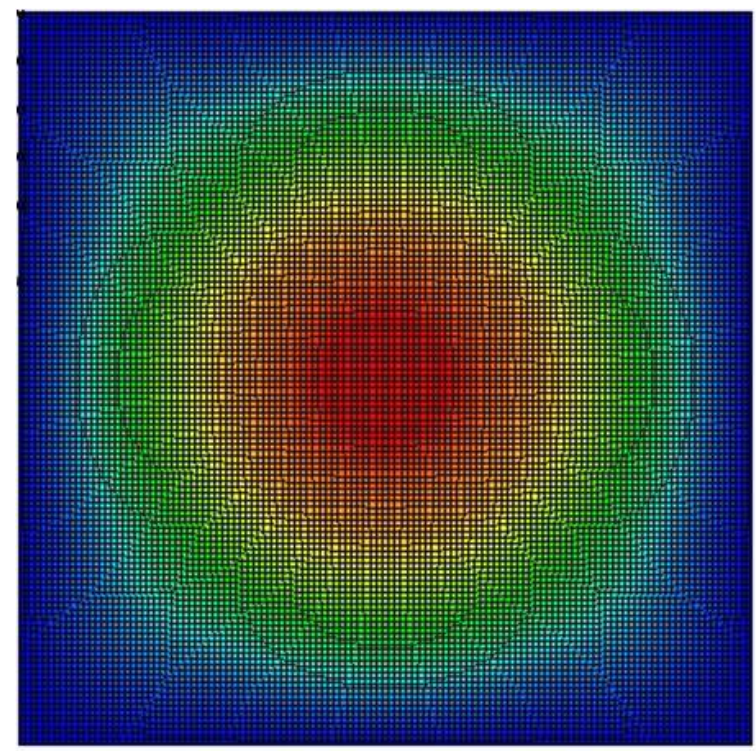

(a)

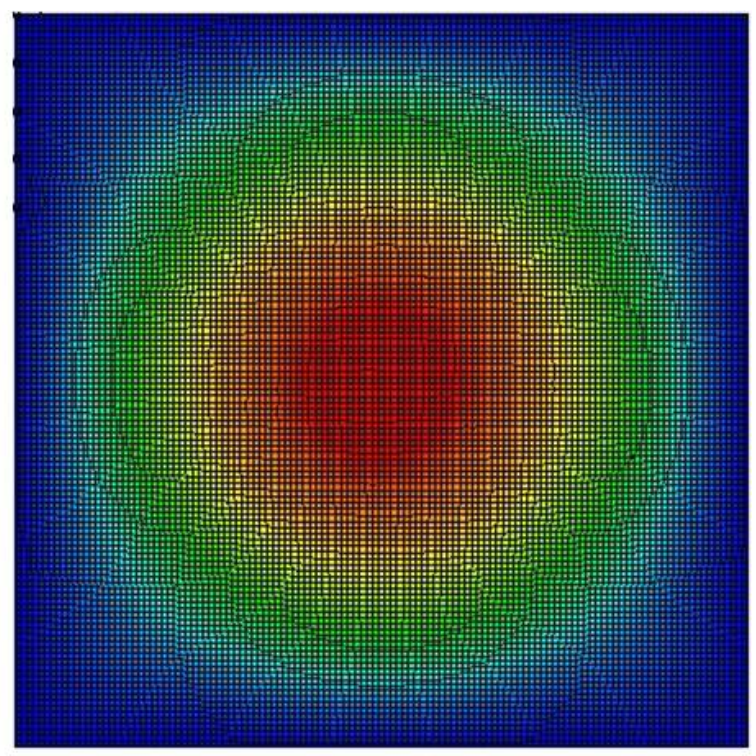

(b)

Figure 15: Displacement Contour Comparison at 500 microseconds, (a) ALE3D, (b) Paradyn SPH 
Peak displacement appears to have been reached by 750 microseconds (Figure 16) and by 1 millisecond the plate appears to be rebounding (Figure 17). Both codes and thus both methods of modeling the HE prove to be similarly loading the plate.

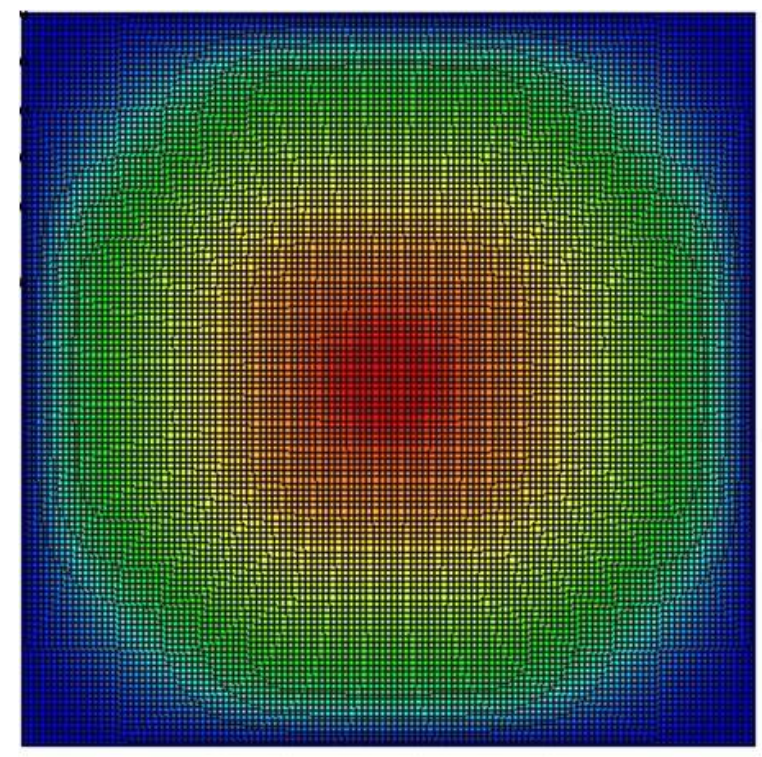

(a)

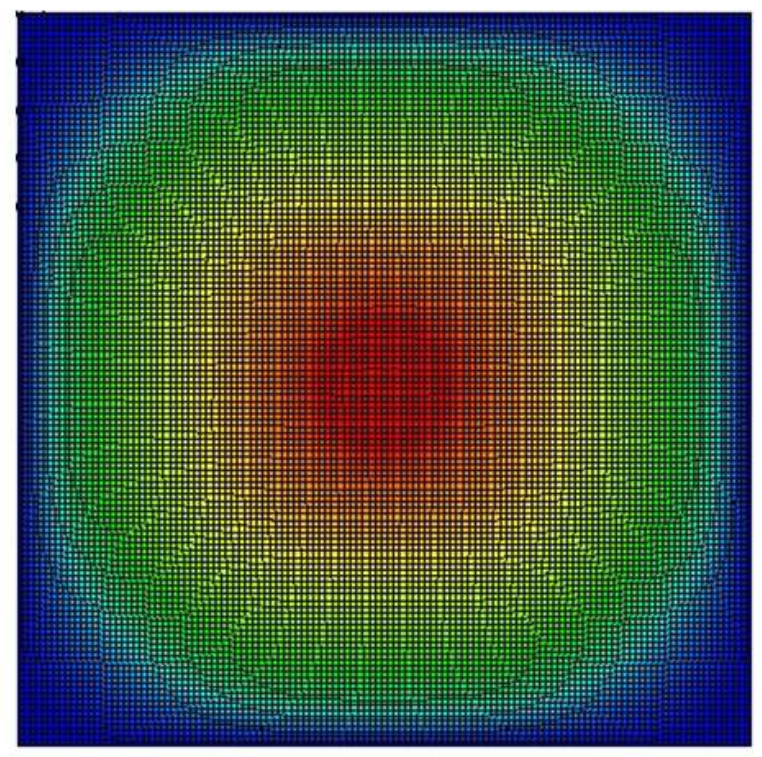

(b)

Figure 16: Displacement Contour Comparison at 750 microseconds, (a) ALE3D, (b) Paradyn $\mathrm{SPH}$

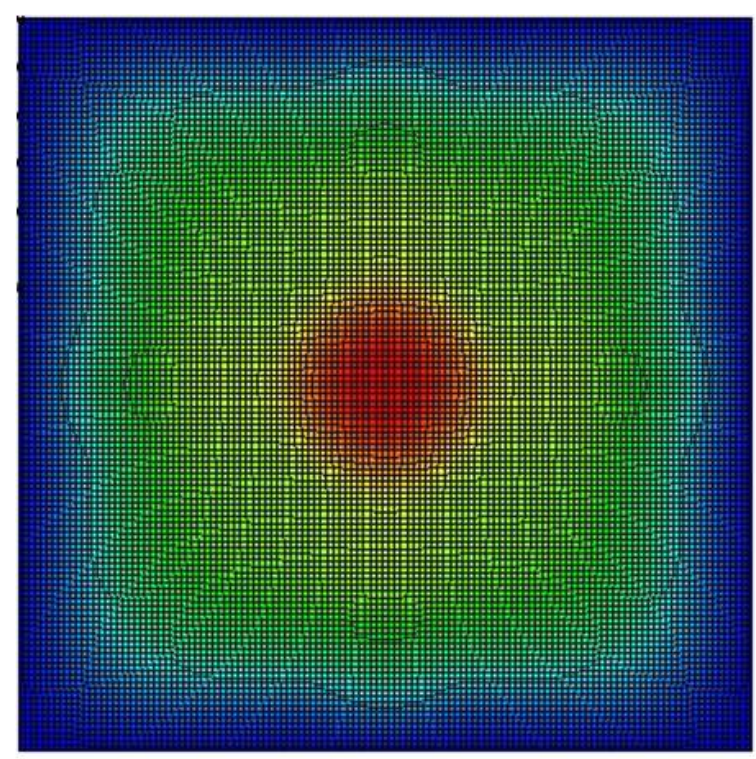

(a)

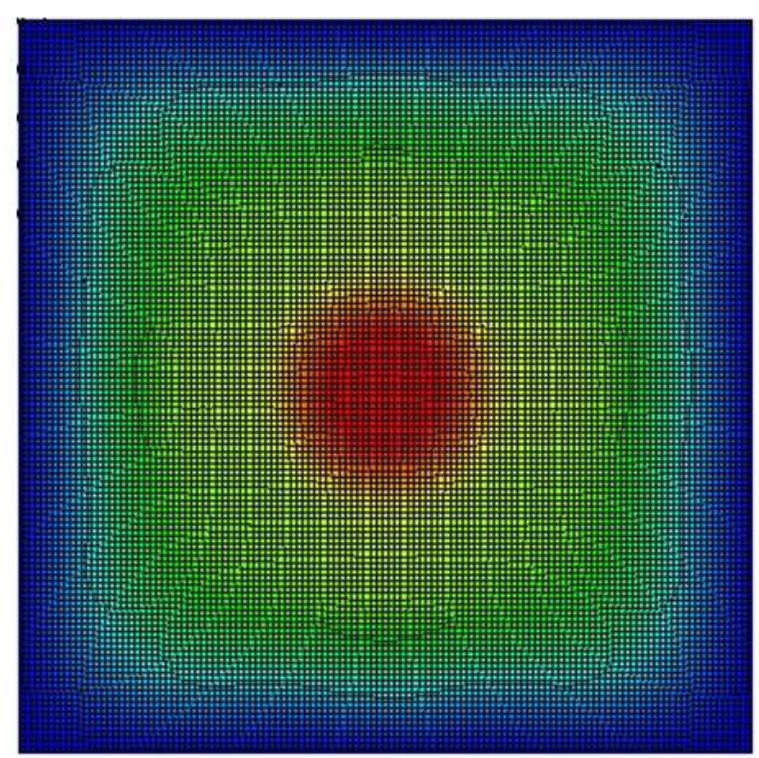

(b)

Figure 17: Displacement Contour Comparison at 1 millisecond, (a) ALE3D, (b) Paradyn SPH 
Displacement time history plots are generated for the plate center and seven centimeters left of center and compared between the three simulations: ALE3D, simplified handshake method described in an earlier section, and the SPH (Figure 18). Figures 15-17 show similar displacement distributions over the surface of the plate, and these plots provide a good assessment of the temporal response.

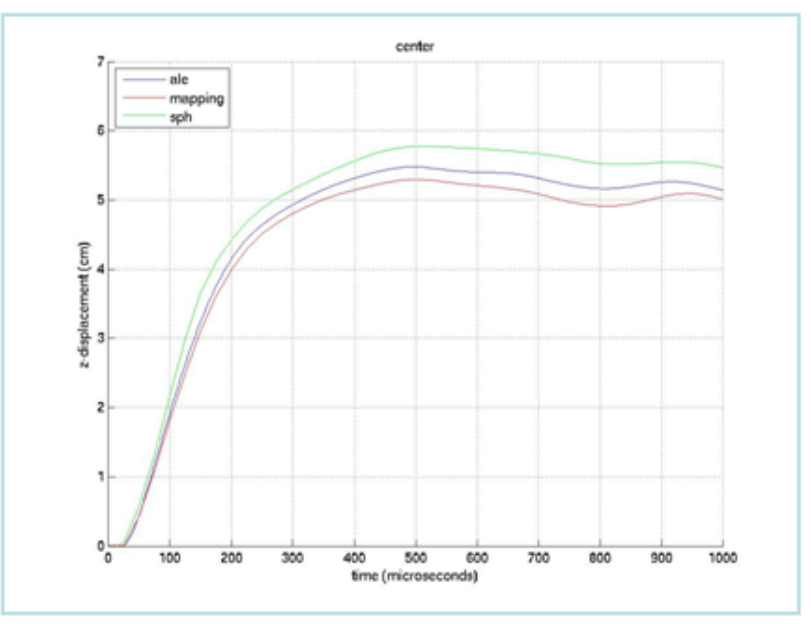

(a)

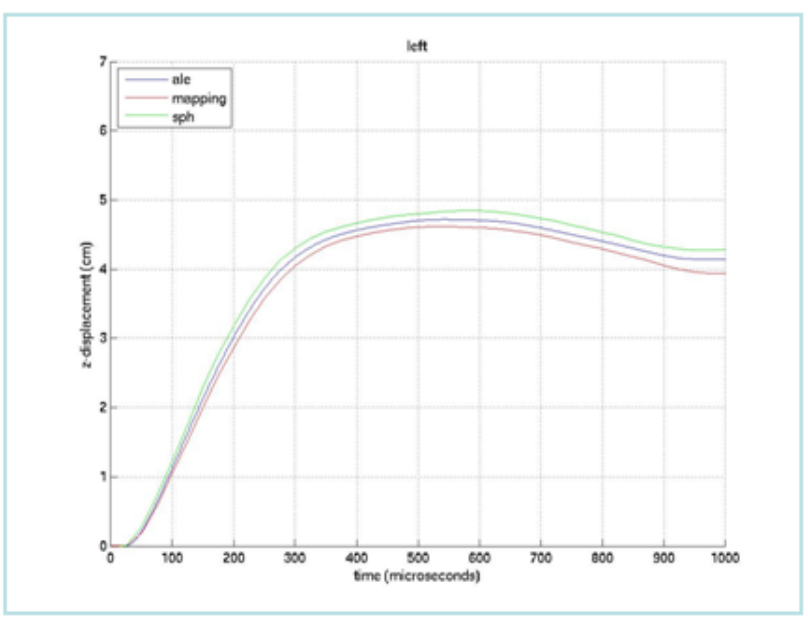

(b)

Figure 18: Displacement Time History Comparison, (a) Plate Center, (b) 7cm Left of Plate Center

\section{Future Work}

By no means can this be considered a complete study of the SPH capability of modeling a high explosive event. This was a specific study with a specific set of test parameters, and needs to be assessed with more scenarios over time.

Based on the work presented in this paper, it is unknown if a structure with smaller elements requires smaller SPH spacing, or rather if a structure made of larger elements would suffice with large SPH spacing for that matter.

With a smaller standoff there is risk of insufficient time before impact for the chemistry to release the full energy of the explosive. Also, with a higher standoff it is unknown what size of $\mathrm{SPH}$ cloud is required. Figures 18 shows how the cloud size affects how much of the pressure front is actually captured, as well as how much of the surrounding air is available to react with. 


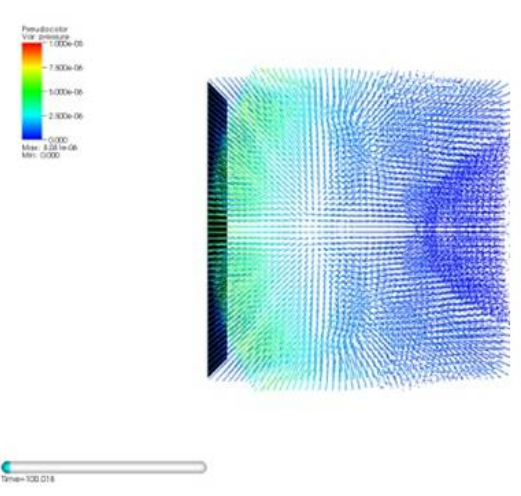

(a)

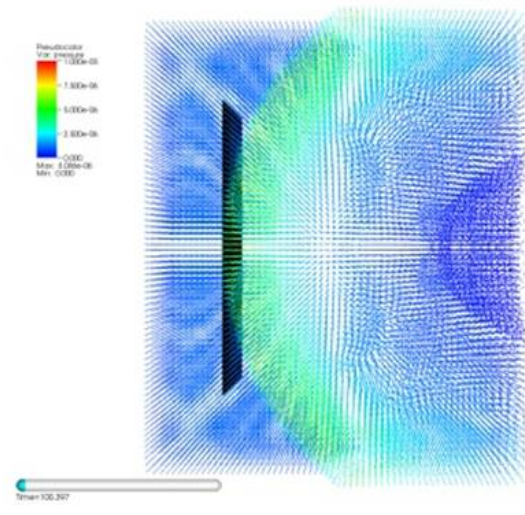

(b)

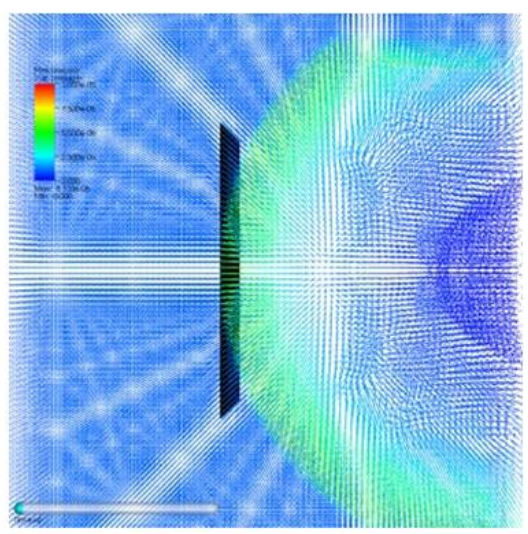

(c)

Figure 19: Large Standoff Example Initial SPH Plot, (a) Small SPH Cloud, (b) Medium SPH Cloud, (c) Large SPH Cloud

Figure 19 shows the deformation of the plate some time after impact. It is clear that the cloud size has a significant effect on the plate response. This could either be the result of the air behind the plate that must be pushed away in order to displace the plate, the effect of the pressure wrapping around the side of the plate, or a combination of the two.

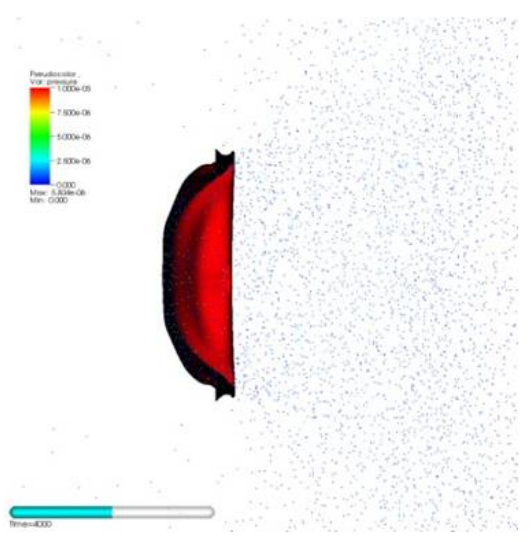

(a)

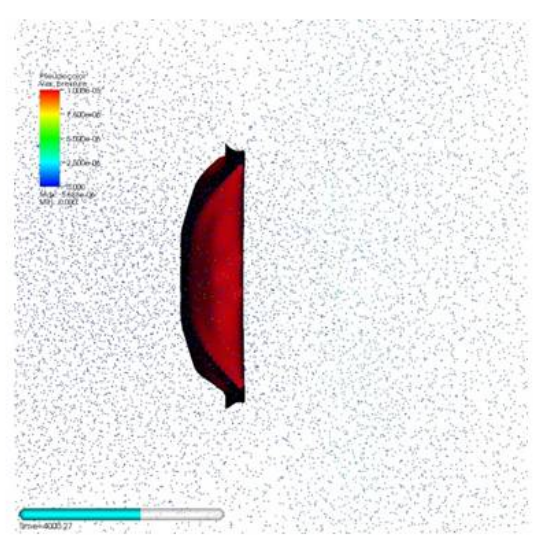

(b)

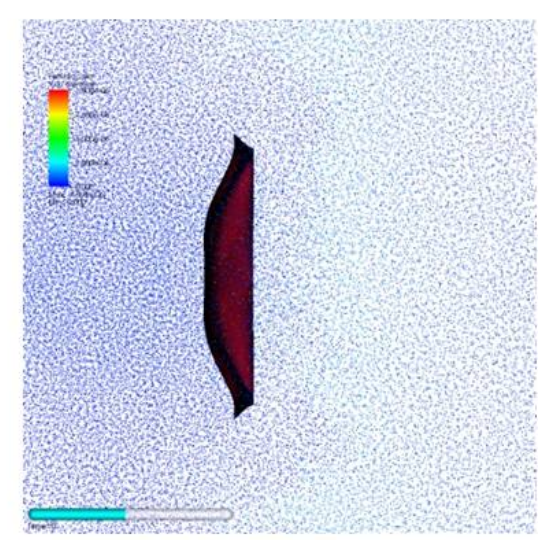

(c)

Figure 20: Large Standoff Example, Plate Deformation, (a) Small SPH Cloud, (b) Medium SPH Cloud, (c) Large SPH Cloud

Because the particle density is reduced as the particles expand, a particle birth method has enormous potential in this type of problem. This is not an easy idea to implement but may come up in the future if the demand exists.

A study should be conducted regarding the number of processors to run the code. An efficient relationship between number of processor and number of SPH particles has not been 
investigated. It would seem that more and more processors would at some point be detrimental as the computers have to work too hard to communicate with one another.

Finally it is of considerable interest if some other medium can be modeled by the particles other than air and the explosive products. Water, foam, or some other kind of low strength material could be well represented, but more studies should be conducted to validate the capability.

\section{Conclusions}

SPH has proven to be a quality meshless technique for modeling explosive materials and the surrounding air, and handling the interactions with a nearby structure. This work has barely scratched the surface for what could be a broad scope of future work. The solution to the strain localization revealed itself as required particle spacing relative to the structural mesh, and this knowledge could be useful to any future work in the area.

As far as the current fully detailed structure, this work has opened the door for full coupling when it previously was not possible, and the project can proceed because of these findings.

\section{Disclaimer \& Auspices}

This report was prepared as an account of work sponsored by an agency of the United States Government. Neither the United States Government, nor any agency thereof, nor any of their employees, nor any of their contractors, subcontractors, or their employees, make any warranty, express or implied, or assume any legal liability or responsibility for the accuracy, completeness, or usefulness of any information, apparatus, product, or process disclosed, or represent that its use would not infringe privately owned rights. Reference herein to any specific commercial product, process, or service by trade name, trademark, manufacturer, or otherwise, does not necessarily constitute or imply its endorsement, recommendation, or favoring by the United States Government, any agency thereof, or any of their contractors or subcontractors. The views and opinions expressed herein do not necessarily state or reflect those of the United States Government, any agency thereof, or any of their contractors. This work was performed under the auspices of the U.S. Department of Energy by Lawrence Livermore National Laboratory under Contract DE-AC52-07NA27344. 


\section{References}

Glascoe, L., C. Noble, L. Leininger, and J. Margraf, ALE3D Airblast Simulations for Disk and Spherical Charges,(SSI) NEWTON Report 2008-1004, for the NEXESS Center, Lawrence Livermore National Laboratory (LLNL-TR-400788), April 25, 2008.

Morris, J., E. Kokko, D. Faux, L. McMichael, ParaDyn: Addition of SPH Framework to Account for Fluid-Structural Interaction During Hypervelocity Impact Events, for $10^{\text {th }}$ US National Congress on Computational Mechanics, Lawrence Livermore National Laboratory (LLNL-PRES-414461), July, 2009.

Nichols III, A. L., ed., Users Manual for ALE3D, An Arbitrary Lagrange/Eulerian 2D and 3D Code System, Volumes 1 and 2, UCRL-MA-152204 Rev. 7, (Export Controlled) Lawrence Livermore National Laboratory, 2008.

Zywicz, E., J. Lin, User Manual for DYNA3D: A Nonlinear, Explicit, Three-Dimensional Finite Element Code for Solid and Structural Mechanics, (Export Controlled) Lawrence Livermore National Laboratory, July, 2011. 